\title{
Effects of Processing Conditions on Biocrude Yields from Fast Hydrothermal Liquefaction of Microalgae
}

\author{
Julia L. Faeth ${ }^{\mathrm{a}}$, Phillip E. Savage $\mathrm{a}^{\mathrm{a}, \mathrm{b}, *}$ \\ ${ }^{a}$ Department of Chemical Engineering, University of Michigan, 2300 Hayward Street, \\ 3074 H.H. Dow Building, Ann Arbor, Michigan 48109, United States \\ ${ }^{b}$ Department of Chemical Engineering, The Pennsylvania State University, 160 Fenske \\ Lab, University Park, Pennsylvania 16802, United States
}

\begin{abstract}
This study investigated the effects of algae species, reaction time, and reactor loading on the biocrude yield from fast hydrothermal liquefaction (HTL) of microalgae. Fast HTL reaction times were always less than 2 min and employed rapid heating and nonisothermal conditions. The highest biocrude yield obtained was $67 \pm 5$ wt $\%$ (dry basis). With all other process variables fixed, increasing the reaction time in a $600{ }^{\circ} \mathrm{C}$ sand bath by $15 \mathrm{sec}$ increments led to a rapid increase in biocrude yield between 15 - 45 sec. At longer times, the biocrude yield decreased. Low reactor loadings generally gave higher biocrude yields than did higher loadings. The low reactor loadings may facilitate biocrude production by facilitating cell rupture and/or increasing the effective concentration of algal cells in the hot, compressed water in the reactor.
\end{abstract}

Keywords: Biofuel, Hydrothermal Liquefaction, Microalgae

\footnotetext{
*Corresponding author. Tel.: +1-814-867-5876; fax: +1-814-865-7846

Email address: psavage@psu.edu (Phillip E. Savage)
} 


\section{Introduction}

$\mathrm{CO}_{2}$ emissions from the combustion of liquid transportation fuels contribute to anthropogenic global climate change. Renewable fuels from biomass may reduce the net $\mathrm{CO}_{2}$ emissions from a life cycle perspective, depending on factors such as the energy intensity of biomass cultivation, harvesting/collection, and conversion processes. Hydrothermal liquefaction (HTL) of microalgae is a biomass conversion process that could reduce life cycle $\mathrm{CO}_{2}$ emissions compared to petroleum-derived fuels, especially when algae cultivation is coupled with a wastewater treatment plant (Fortier et al., 2014). HTL also has the advantage of avoiding the energy-intensive biomass drying steps that are required for many other biofuel production processes (Marcilla et al., 2013). Briefly, HTL accomplishes biocrude production by the decomposition of biomolecules in hot compressed water via the combined action of elevated temperature, elevated pressure, and hydrolytic attack. HTL of microalgae has been the focus of several reviews (Barreiro et al., 2013a; Chow et al., 2013; Marcilla et al., 2013; Tian et al., 2014).

A large majority of the algal HTL literature examines hydrothermal reactions at $300-350{ }^{\circ} \mathrm{C}$ with reaction times on the order of tens of minutes. Faeth et al. recently described a variation on the HTL process, termed fast HTL (Faeth et al., 2013), that requires just a short reaction time (up to about $2 \mathrm{~min}$ ) and rapid heating rates $\left(150-300{ }^{\circ} \mathrm{C} / \mathrm{min}\right)$. This rapid heating facilitates the production of biocrude from microalgae with yields comparable to or higher than those from conventional isothermal HTL (Faeth et al., 2013). More recently, there have been investigations of fast HTL of macroalgae (Bach et al., 2014), HTL of microalgae for short residence times in a 
continuous flow system (Jazrawi et al., 2013), and fast HTL of bacteria and yeast (Valdez et al., 2014). These studies demonstrated the robustness of this fast HTL approach but much about this promising HTL variation remains unknown. To fill some of these gaps, we explore herein fast HTL of four species of microalgae: Nannochloropsis sp., Chorella vulgaris, Botryococcus braunii, and Neochloris oleoabundans and the effects of different aspects of reactor loading and reaction time on fast HTL of Nannochloropsis sp.

\section{Materials and Methods}

Materials used in this work are identical to those described previously (Faeth et al., 2013) and were purchased and/or constructed in the same manner. Briefly, mini batch reactors were constructed from 316 stainless steel with an internal volume of $1.67 \mathrm{~mL}$. Most data reported herein describe batch reactions using Nannochloropsis sp. from Reed Mariculture, Inc., although we also compare results from HTL of Nannochloropsis sp. to those from Chorella vulgaris, Botryococcus braunii, and Neochloris oleoabundans cultivated at the University of Dayton Research Institute (UDRI). Batch mini-reactors were loaded with algae and water (Please see Tables S1 - S5 in the Supporting Information for loading details) and sealed to $45 \mathrm{ft}$-lbs using a torque wrench. Sealed reactors, accompanied by an empty thermocoupleequipped dummy reactor, were submerged in a fluidized sand bath preheated to $600{ }^{\circ} \mathrm{C}$ for fast $\mathrm{HTL}$, and $350{ }^{\circ} \mathrm{C}$ for isothermal HTL. The reaction time was defined to begin at the instant reactors were submerged and to end at the instant reactors were quenched in a cold water bath.

Cooled reactors were opened and the products collected by pouring the 
reactor contents into a glass centrifuge tube, rinsing the reactor with $9 \mathrm{~mL}$ of dichloromethane, and collecting these rinsings in the same test tube. The biocrude (organic phase), solid, and aqueous phase products were separated with centrifugation and manual pipeting as described previously (Valdez et al., 2012; Faeth et al., 2013; Roberts et al., 2013; Barreiro et al., 2013b). Once separated, the biocrude products were dried under $\mathrm{N}_{2}$. After drying, the masses of the biocrude products were recorded. We report biocrude yields as the mass of biocrude produced divided by the mass of dry algae loaded into the reactor.

\section{Results and Discussion}

This section presents the effects of algae species, reaction time, and reactor loading on biocrude production from HTL of microalgae. The first subsection includes four species of microalgae, but all subsequent subsections focus on results from the HTL of Nannochloropsis sp.

\subsection{Algae Species}

Figure 1 depicts biocrude yields (in wt $\%$ on a dry basis) from fast (600 ${ }^{\circ} \mathrm{C}$ sand bath for $\left.1 \mathrm{~min}\right)$ and isothermal $\left(350{ }^{\circ} \mathrm{C}\right.$ sand bath for $\left.60 \mathrm{~min}\right) \mathrm{HTL}$ of four species of microalgae. The standard error from at least three replicates of each reaction condition is represented by the error bars on this figure and all others in this work, unless otherwise indicated. The biocrude yield produced from isothermal HTL of Nannochloropsis sp. depicted in Figure 1 is taken from previous work (Faeth et al., 2013) and is based on a single replicate. 
For all species of microalgae examined in the present work, fast HTL produces biocrude yields comparable to those from isothermal HTL. Fast HTL of Botryococcus braunii and Nannocholoropsis sp. produced more biocrude than did isothermal HTL. Along with the previous work investigating fast HTL of bacteria and yeast (Valdez et al., 2014), these results are a testament to the robustness of fast HTL as a biomass conversion process for microorganisms. The differences in biocrude yields between different algae species likely reflect the differences in biochemical composition (i.e., protein, lipid, carbohydrate, ash) and/or cellular structure (i.e. cell wall strength, cell shape) between species.

\subsection{Reaction Time}

To examine the effects of reaction time in greater detail, we conducted fast HTL experiments with reaction times ranging from $0.25-2 \mathrm{~min}$ with a sand-bath temperature of $600{ }^{\circ} \mathrm{C}, 15 \mathrm{wt} \%$ solids in slurry and 11 volume $\%$ water loading (meaning that $11 \%$ of the reactor volume is occupied by water at room temperature). At these conditions, the biocrude yields increase rapidly between 15 - 45 sec and decrease with further increases in reaction time. The highest biocrude yield observed in this set of reactions is $55 \mathrm{wt}$ \%. This biocrude yield is obtained after only $45 \mathrm{sec}$ in the $600{ }^{\circ} \mathrm{C}$ sand bath. At this reaction time, the empty, dummy reactor temperature was $188^{\circ} \mathrm{C}$, which provided an estimate of the temperature in the HTL reactor. The rapid increase in biocrude yield during this $30 \mathrm{sec}$ interval might be due to cell breakage occurring over this same interval. Cell breakage, which could occur at different characteristic temperatures for different algal species (Garcia Alba et al., 2012) and possibly be accelerated by rapid heat- 
ing, would release triglycerides and other biomolecules that may contribute to high biocrude yields. Differences in thermal conductivity between the intracellular water and bulk water in the reactor may facilitate development of pressure differentials under rapid heating to induce cell rupture. Decreasing biocrude yields with increasing reaction time beyond $0.75 \mathrm{~min}$ is likely the result of hydrothermal degradation of cellular components to water-soluble and gaseous products, with the latter becoming more significant at temperatures exceeding the critical point of water $\left(374^{\circ} \mathrm{C}\right)$.

\subsection{Reactor Loading}

There are three ways to systematically vary the contents of the mini batch reactor during fast HTL. One way is to use a fixed amount of water in each reactor, but vary the amount of algae. A second way is to load different amounts of algae slurry with a fixed solids content (e.g., 15 wt \%). The third way is to use a fixed amount of dry algal biomass in each reactor but vary the amount of water added. This section examines each of these methods.

These variations in reactor loading will be reported and discussed in terms of two key variables: the percentage of the reactor volume occupied at room temperature by the water loaded into the reactor (hereafter referred to as volume $\%$ water) and the solids content (wt \%) in the algae slurry present in the reactor at $\mathrm{t}=0$. These two variables control the "effective concentration" of algae solids in hot compressed water at a given reaction temperature. As the reactor is heated, some of the liquid water must vaporize to maintain the saturation pressure, which leaves less water in the liquid phase. The lower the water loading, the higher the relative amount of water that must vaporize. 
We define the effective concentration (wt \%) of algae as the mass of dry algae in the reactor divided by the mass of algae plus the mass of liquid water in the reactor at a given temperature. The mass of liquid water in the reactor at a given temperature was calculated using mass balances and the steam tables (Haar et al., 1984). Figure 2 depicts how the effective concentration at $266{ }^{\circ} \mathrm{C}$ varies with the vol \% water loading and solids content (wt \%). The numbers superimposed on Figure 2 represent the biocrude yields from HTL reactions encompassing all three variations on reactor loading described previously. The $266{ }^{\circ} \mathrm{C}$ temperature represents the temperature a dummy reactor typically reaches after $1 \mathrm{~min}$ in a $600{ }^{\circ} \mathrm{C}$ sand bath, based on the average of all temperature profiles collected in this study.

At high vol \% water loading and high solids content, the algae concentration at reaction conditions is nearly the same as the initial wt \% loading. At low water loadings, however, the effective concentration can depart significantly from the initial wt \% loading. For example, an initial loading of 25 wt \% slurry that provides enough water to fill just $10 \%$ of the reactor at room temperature will have an effective concentration of about $30 \mathrm{wt} \%$ when the reactor reaches $266{ }^{\circ} \mathrm{C}$.

\subsubsection{Fixed Water Loading}

Figure 3 illustrates how biocrude yields from fast HTL $\left(600{ }^{\circ} \mathrm{C}\right.$ for $\left.1 \mathrm{~min}\right)$ of algae change with respect to the algal solids content (wt \%) when a fixed amount of water (either 11 or $30 \mathrm{vol} \%$ ), was added to each reactor. The mass of algae added to each reactor was adjusted to obtain the desired wt $\%$ of dry algae solids content.

At 11 vol \% water in Figure 3, biocrude yields decrease from 67 wt \% to 
43 wt \% as the solids content of the algal slurry increases from 5 to 25 wt \% solids. The highest biocrude yield with $30 \mathrm{vol} \%$ water is also observed after fast HTL of a reaction mixture containing $5 \mathrm{wt} \%$ algae solids. However, fast HTL reactions with $30 \mathrm{vol} \%$ water resulted in all biocrude yields being within $5 \mathrm{wt} \%$ of the maximum biocrude yield for all solids contents examined. This difference in biocrude production between high and low water loadings could possibly be explained by changes in effective concentration.

\subsubsection{Fixed Solids Content}

To manipulate the vol \% water in the reactor and maintain fixed solids content, the mass of both algae and water were varied. As the vol \% water increases, the biocrude yields decrease slightly. As indicated in Figure 2, the effective concentration of algal biomass in the reactor at reaction conditions increases as mass loadings (and vol \% water) decrease. Effective concentrations for the reaction conditions with fixed solids content increase from 15.4 to 18.8 wt $\%$ as the vol \% water decreases from $60 \%$ to $10 \%$. If the reactions of algal biomass to produce biocrude are greater than zero-order, an increase in the effective concentration of the reactant(s) in the liquid phase would increase the rate of biocrude production. It is also possible that cell breakage is facilitated by the low loading reaction environments, as discussed previously.

\subsubsection{Fixed Biomass Loading}

For a constant biomass loading, as the water loading decreases, the effective concentration increases. Based on experiments with a constant biomass loading, biocrude yields generally increase as the effective concentration in- 
creases. This observation further supports the idea that the effective concentration of algal biomass in the reaction mixture has an effect on biocrude production.

\subsubsection{All Reactor Loading Variables}

Figure 2 depicts the biocrude yields with respect to both vol \% water and algal slurry solids content for all reactor loading experiments discussed above. Generally, biocrude yields increase with increasing effective concentration for a given slurry solids content (this also corresponds to a decrease in vol $\%$ water). For a given vol \% water, the correlation between biocrude yields and effective concentration is less clear. Further research may be required to elucidate more completely how and why the effects of reactor loading influence biocrude yields.

\subsection{Summary of Results}

Fast HTL produced biocrude from four different species of microalgae with yields comparable to those available from isothermal HTL, further illustrating that fast HTL is a robust process. The processes that produce biocrude from microalgal biomass are very fast. The highest biocrude yields from non-isothermal HTL of a 15 wt \% algal slurry with 11 vol \% water were obtained at times between 0.75 - 1.0 min (using a $600{ }^{\circ} \mathrm{C}$ sand-bath set-point temperature). Such rapid conversion might arise from cell breakage that occurs at characteristic temperatures, possibly accelerated by high heating rates and thermal conductivity differences between intra- and extracellular material which could induce pressure differentials across cell walls.

Different aspects of reactor loading affect biocrude yields. Fast HTL of 
mixtures with low solid contents can produce high biocrude yields when the total mass in the reactor is low. Specifically, we observed the highest biocrude yield of $67 \mathrm{wt} \%$ after HTL of a $5 \mathrm{wt} \%$ algal slurry, $11 \mathrm{vol} \%$ water in a 1 min in a $600{ }^{\circ} \mathrm{C}$ sand bath. In contrast, variation in algae solids content in reactions with high total mass loadings (high vol \% water) does not appear to have a clear impact on biocrude production via fast HTL. This observed increase in yield with low water loading and low solids content could be due, at least in part, to an increase in the effective concentration of algae cells in the liquid phase of the reaction, relative to an otherwise identical reaction with high total mass loading.

Manipulation of reactor loading by fixing the solids wt \% content and adjusting the total mass of the reaction mixture revealed a decrease in biocrude yields with increasing mass. Further, biocrude yields decreased as the mass of water increased for fast HTL reactions with a fixed amount of dry algae biomass. Both of these trends reinforce the idea that increased effective concentration of algae in the liquid phase during rapid heating may facilitate biocrude production. Additionally, the volume $\%$ water has a greater effect on biocrude yields than does the wt $\%$ of dry algae solids present in the slurry.

\section{Conclusions}

This study provides the most detailed examination to date of how fast HTL processing conditions (including biomass feedstock, reaction time, and reactor loading) affect biocrude yield. The results discussed in this work suggest that physical phenomena, in addition to chemical reactions, may 
contribute to the improved biocrude yields obtained via fast HTL under certain operating conditions, and that further research in this area is needed to better understand and model the fast HTL process.

\section{Acknowledgement}

Nick Garza and Hao Chen provided experimental assistance. Dr. Jerome Servaites and Dr. Sukhjinder Sidhu of the University of Dayton Research Institute graciously provided algal biomass for this research. The National Science Foundation (Grant EFRI-0937992), the University of Michigan Rackham Graduate School, and the University of Michigan College of Engineering provided financial support. Julia Faeth acknowledges that this material is based on work supported by the National Science Foundation Graduate Student Research Fellowship under Grant DGE 1256260.

\section{Supplementary Information}

The Supporting Information provides details regarding the algae and water loadings for all experiments. Table S1 contains the loadings for the data presented in Figure 1, Table S2 contains the loadings for the reaction time experiements (not depicted in a figure), Table S3 contains the loadings for Figure 3 (also depicted in Figure 2), Table S4 contains the loadings for the fixed solids content experiments (depicted in Figure 2, along with other experiments), and Table S5 contains the loadings for fixed biomass loading experiments (depicted in Figure 2, along with other experiments).

Bach, Q.V., Sillero, M.V., Tran, K.Q., Skjermo, J., 2014. Fast hydrothermal liquefaction of a Norwegian macro- 
alga: Screening tests. Algal Research 6, 271-276. URL: http://linkinghub.elsevier.com/retrieve/pii/S2211926414000538, doi:10.1016/j.algal.2014.05.009.

Barreiro, D.L., Prins, W., Ronsse, F., Brilman, W., 2013a. Hydrothermal liquefaction (HTL) of microalgae for biofuel production: State of the art review and future prospects. Biomass and Bioenergy 53, 113-127. URL: http://linkinghub.elsevier.com/retrieve/pii/S0961953412005272, doi:10.1016/j.biombioe.2012.12.029.

Barreiro, D.L., Zamalloa, C., Boon, N., Vyverman, W., Ronsse, F., Brilman, W., Prins, W., 2013b. Influence of strain-specific parameters on hydrothermal liquefaction of microalgae. Bioresource Technology 146, 463-71. URL: http://www.ncbi.nlm.nih.gov/pubmed/23958678, doi:10.1016/j.biortech.2013.07.123.

Chow, M.C., Jackson, W.R., Cha, A.L., Marshall, M., 2013. Thermal Treatment of Algae for Production of Biofuel. Energy \& Fuels 27, 19261950.

Faeth, J.L., Valdez, P.J., Savage, P.E., 2013. Fast Hydrothermal Liquefaction of Nannochloropsis sp. To Produce Biocrude. Energy \& Fuels 27, 1391-1398. URL: http://pubs.acs.org/doi/abs/10.1021/ef301925d, doi:10.1021/ef301925d.

Fortier, M.O.P., Roberts, G.W., Stagg-Williams, S.M., Sturm, B.S.M., 2014. Life cycle assessment of bio-jet fuel from hydrothermal liquefaction of microalgae. Applied Energy 122, 73-82. URL: 
http://linkinghub.elsevier.com/retrieve/pii/S030626191400107X, doi:10.1016/j.apenergy.2014.01.077.

Garcia Alba, L., Torri, C., Samorì, C., Spek, J.V.D., Fabbri, D., Kersten, S.R.A., Brilman, D.W.F.W., 2012. Hydrothermal Treatment (HTT) of Microalgae: Evaluation of the Process As Conversion Method in an Algae Biorefinery Concept. Energy \& Fuels 26, 642-657.

Haar, L., Gallagher, J.S., Kell, G.S., 1984. NBS/NRC Steam Tables. Hemisphere Publishing Corporation, Washington, DC.

Jazrawi, C., Biller, P., Ross, A.B., Montoya, A., Maschmeyer, T., Haynes, B.S., 2013. Pilot plant testing of continuous hydrothermal liquefaction of microalgae. Algal Research 2, 268-277. URL: http://linkinghub.elsevier.com/retrieve/pii/S2211926413000532, doi:10.1016/j.algal.2013.04.006.

Marcilla, A., Catalá, L., García-Quesada, J., Valdés, F., Hernández, M., 2013. A review of thermochemical conversion of microalgae. Renewable and Sustainable Energy Reviews 27, 11-19. URL: http://linkinghub.elsevier.com/retrieve/pii/S1364032113004097, doi:10.1016/j.rser.2013.06.032.

Roberts, G.W., Fortier, M.O.P., Sturm, B.S.M., Stagg-Williams, S.M., 2013. Promising Pathway for Algal Biofuels through Wastewater Cultivation and Hydrothermal Conversion. Energy \& Fuels 27, 857867.

Tian, C., Li, B., Liu, Z., Zhang, Y., Lu, H., 2014. Hydrothermal liquefaction for algal biorefinery: A critical review. Re- 
288 newable and Sustainable Energy Reviews 38, 933-950. URL:

289 http://linkinghub.elsevier.com/retrieve/pii/S1364032114004821,

290 doi:10.1016/j.rser.2014.07.030.

${ }_{291}$ Valdez, P.J., Nelson, M.C., Faeth, J.L., Wang, H.Y., Lin, X.N., Savage, P.E., 292 2014. Hydrothermal Liquefaction of Bacteria and Yeast Monocultures. ${ }_{293}$ Energy \& Fuels 28, 67-75.

${ }_{294}$ Valdez, P.J., Nelson, M.C., Wang, H.Y., Lin, X.N., Savage, P.E., 2012. Hy295 drothermal liquefaction of Nannochloropsis sp.: Systematic study of pro296 cess variables and analysis of the product fractions. Biomass \& Bioenergy $297 \quad 46,317-331$. 


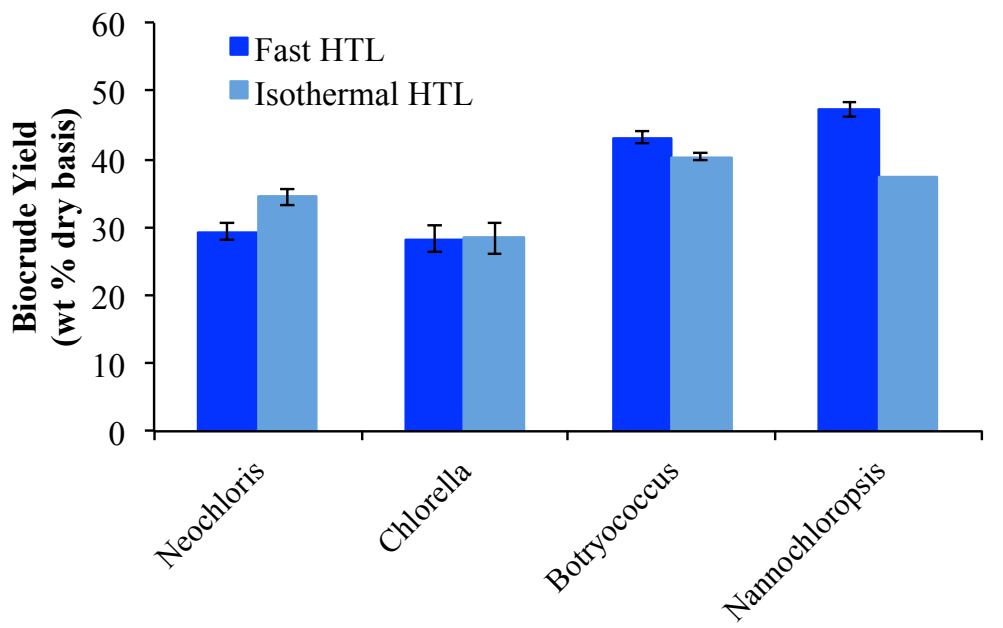

Figure 1: Biocrude Yields from Fast $\left(600{ }^{\circ} \mathrm{C}\right.$ sand bath, 1 min, 30 vol $\%$ water loading) and Isothermal $\left(350^{\circ} \mathrm{C}\right.$ sand bath, $60 \mathrm{~min}, 55 \mathrm{vol} \%$ water loading) HTL of Different Algae Species, 15 wt \% solids in slurry 


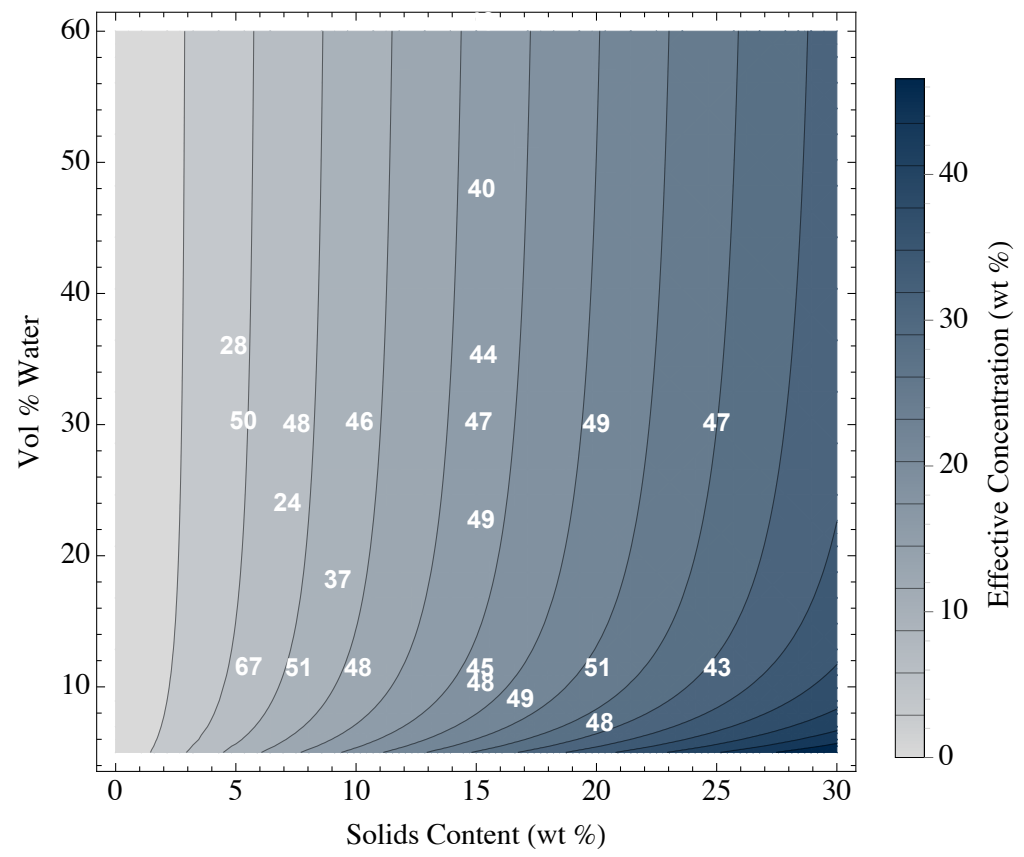

Figure 2: Biocrude yields (wt \% dry basis, in text) from fast HTL at different reactor loadings at different vol $\%$ water and slurry solid contents. Sand-bath temperature $=600$ ${ }^{\circ} \mathrm{C}, 1 \min$ 


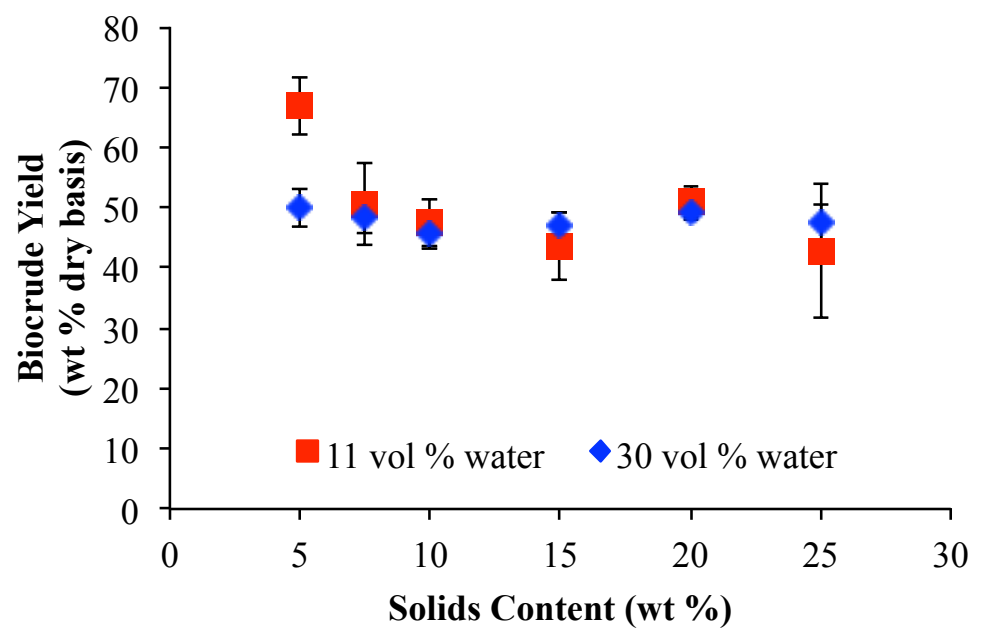

Figure 3: Biocrude yields from fast HTL (sand-bath temperature $=600{ }^{\circ} \mathrm{C}, 1 \mathrm{~min}$ ) with different slurry solid contents at low (11 vol \%) and high (30 vol \%) water loading 